\title{
Initiation of student-TA interactions in tutorials
}

\author{
Rachel E. Scherr, Rosemary S. Russ, Thomas J. Bing, and Raymond A. Hodges \\ Physics Education Research Group, Department of Physics, University of Maryland, College Park, Maryland 20742, USA
}

(Received 30 March 2006; published 14 December 2006)

\begin{abstract}
At the University of Maryland we videotaped several semesters of tutorials as part of a large research project. A particular research task required us to locate examples of students calling the teaching assistants (TAs) over for assistance with a physics question. To our surprise, examples of this kind of interaction were difficult to find. We undertook a systematic study of TA-student interactions in tutorial: In particular, how are the interactions initiated? Do the students call the TA over for help with a particular issue, does the TA stop by spontaneously, or does the worksheet require a discussion with the TA at that point? The initiation of the interaction is of particular interest because it provides evidence of the motivation for and purpose of the interaction. This paper presents the results of that systematic investigation. We discovered that the majority of student-TA interactions in tutorial are initiated by teaching assistants, confirmed our initial observation that relatively few interactions are initiated by students, and found, further, that even fewer interactions are worksheet initiated. Perhaps most importantly, we found that our sense of who initiates tutorial interactions-based on extensive but informal observations-is not necessarily accurate. We need systematic investigations to uncover the reality of our classroom experiences.
\end{abstract}

DOI: 10.1103/PhysRevSTPER.2.020108

PACS number(s): 01.40.Fk, 01.40.gb, 01.40.J-

\section{INTRODUCTION}

As experienced tutorial instructors and developers, we are frequently called on to describe the role of teaching assistants (TAs) in the tutorial classroom. Usually we respond that as students work together on structured conceptual worksheets, a TA moves attentively around the classroom, eavesdropping on the groups of students to loosely monitor their progress. If students reach an impasse in their work together, they may call a TA over for assistance; hopefully, the TA assists them without actually resolving the issue for them, and they resolve it themselves after the TA leaves. There are also checkpoints built into the worksheets to make sure students have certain essentials straightened out before they start new material; every student group is expected to talk to a TA at every checkpoint. TAs are free to stop by a table and start a conversation about an issue they have overheard, but we hope that, mostly, if the students are working well together, the TAs will let them continue to do so. ${ }^{1}$

At the University of Maryland we videotaped several semesters of tutorials as part of a large research project. ${ }^{2}$ A particular research task required us to locate examples of students calling the TAs over for assistance with a physics question. To our surprise, examples of this kind of interaction were difficult to find. Could it be that students rarely initiate interactions with TAs? The possibility concerned us. If students are not initiating interactions, perhaps the tutorials are not as student centered as we imagine. Even more disturbing to us was the possibility that we-as experienced with tutorials as we are-were poorly informed about the realities of the tutorial classroom. Is our promotion of tutorials to students and colleagues, our TA training, and our continued curriculum development well matched to what actually occurs in tutorial sessions?

We decided to undertake a systematic study of TA-student interactions in tutorial. We chose to focus particularly on what brings the TA to the students. Do the students call the
TA over for help with a particular issue, does the TA stop by without an invitation, or does the worksheet require a discussion with the TA at that point? This paper presents the results of that systematic investigation and offers preliminary speculation about what might cause the trends we observe. Future studies will investigate the quantity of student-TA interactions (how often and for how long students and TAs interact) as well as their substance (what the interaction is about).

The initiation of the interaction is of particular interest because it provides evidence of the motivation for and purpose of the interaction. Students are likely to be motivated to initiate interactions because they judge themselves to need assistance or validation. The purpose of the ensuing interaction (at least in their minds) is presumably for them to secure that assistance or validation. TAs, on the other hand, may be motivated to initiate interactions by a sense that students need assistance, a feeling of responsibility to monitor student progress, or curiosity about student thinking, among other possibilities. TA-initiated interactions are more likely to have the purpose of satisfying the TA that the students are making sufficient progress. Worksheet-initiated interactions are most likely to be motivated by obedience to the tutorial's instructions, and are likely to have the purpose of ensuring that students have fulfilled the tutorial's intentions up to the checkpoint.

We discovered that the majority of student-TA interactions in tutorial are initiated by teaching assistants. We confirmed our initial observation that relatively few interactions are initiated by students, and found further that even fewer interactions are worksheet initiated. Perhaps most importantly, we found that our sense of what transpires in tutorial-based on extensive but informal observations-is not necessarily accurate. Partly as a result of this study, we suspect that our perceptions of our teaching may be significantly colored by our wishes, expectations, and prejudices. We need systematic investigations to uncover the reality of our classroom experiences. 


\section{THEORETICAL BACKGROUND}

We had tacit assumptions that student-centered learning environments are desirable, that the tutorial environment is student centered, that student-initiated interactions are an indicator of student centeredness, and that there would therefore be many student-initiated interactions in that environment. This tacit theoretical perspective was contradicted by our initial observation and thereby made explicit.

The components of that tacit perspective, however, are supported by previous research. The idea that studentcentered learning environments are desirable is a commonplace of educational research, and there is even some measure of agreement as to what constitutes such an environment. ${ }^{3}$ The tutorials are promoted as student centered by their developers ${ }^{4}$ and by secondary implementers. ${ }^{5}$ The idea that student-initiated interactions are an indicator of student centeredness is not as commonly articulated; while "active engagement" is accepted as desirable for student learning not only in general but in physics in particular, ${ }^{6}$ students may be actively engaged without initiating interactions with instructors. Research on help-seeking behaviors, however, supports the idea that question asking can be a strategic resource for developing autonomous learning, one that students would do well to employ and teachers would do well to support. ${ }^{7,8}$ For example, students who are more active, engaged, self-regulating, and motivated in their learning are more likely to seek assistance when necessary; ${ }^{9}$ children who prefer challenge and independent mastery are more likely to seek help; ${ }^{10}$ and mastery-oriented college students report seeking help more frequently than those who are motivated primarily by performance goals. ${ }^{11}$

We expected that the tutorial environment, being one in which actively engaged students undertook challenging physics problems in a student-centered learning environment, would be a rich field for observing the question asking that we understood to be desirable. We emphasize that this theoretical perspective shaped our work implicitly. Only in retrospect, when our expectations were contradicted, did our initial assumptions become available to us for examination.

\section{CONTEXT FOR RESEARCH}

This study was conducted in the algebra-based introductory physics course at the University of Maryland. This twosemester sequence is taught to about 500 students in each course in each semester. The class meets for three fiftyminute lectures each week. Each lecture serves 100-200 students, most of whom are junior and senior health and life science majors. Almost all have successfully completed calculus (though most prefer not to use it) and $75 \%$ of them will have worked in a research laboratory by the end of their college careers. Each week, there is a required two-hour laboratory and a one-hour small group session that we run as a tutorial.

Due to the presence of the Physics Education Research Group (PERG), the physics department at the University of Maryland has a long history with tutorial instruction in introductory courses. ${ }^{12}$ The tutorials we use are based on the model pioneered by the Physics Education Group at the Uni- versity of Washington. ${ }^{13}$ In this model, students work in groups of four on carefully designed research-based worksheets. The worksheets lead students to make predictions and compare various lines of reasoning in order to build an understanding of basic concepts. TAs serve as facilitators rather than lecturers; instead of demonstrating problem solutions to students, they engage the students in discussions of difficult concepts. "Checkpoints," explicitly indicated on the worksheets, direct the students to discuss their work so far with a TA to ensure they are making appropriate progress. TAs prepare for each tutorial with a weekly session in which they learn the tutorial material themselves, learn about common student naive conceptions, and learn to facilitate by asking guiding questions and leaving the students to work out most of the difficulties among themselves.

The tutorial worksheets used in this study were developed at the University of Maryland as part of another project. ${ }^{14}$ They differ from University of Washington tutorials in length and in epistemological focus, ${ }^{15}$ but the role of the TAs is similar in the two cases. In the tutorials included in this study, each class section consisted of five groups of four students each, supervised by two TAs.

\section{DATA SOURCE}

As part of another research project, ${ }^{2}$ we had created a library of 380 tutorial videotapes for study. From that library we selected 33 videotaped tutorial hours by the following process:

(i) Out of the four semesters available, we selected a single semester for detailed study (Fall 2002). The primary reason for this choice is that it is in several ways the "best semester" in our records: (a) The students rated the tutorials highly in that semester and considered them important to their learning; (b) most of the TAs were members of the Physics Education Research Group, experienced with tutorial instruction; (c) the lecturers were Physics Education Research (PER) experts who strongly incorporated interactive learning into their lectures and supported the tutorial part of the curriculum; and (d) our videotaping was particularly comprehensive. Our videotapes for that semester document one or two groups for each of eight different sections of 12 different tutorials, for a total of 155 hours. Each videotape documents a single group (the camera does not move).

(ii) From the Fall 2002 set described above, we selected the first seven tutorials of the semester for study. We chose these because they were the best-tested tutorials of the semester and covered basic mechanics topics common to most introductory physics curricula.

(iii) From the collection of tapes of those seven tutorials from Fall 2002, we selected five student groups that earlier observers (for a different project) had identified as being "watchable:" They were consistently on task and talked to each other frequently. ${ }^{16}$ We represent the student groups with colors (red, blue, green, orange, and yellow). The five student groups appear in four different sections (labeled A-D).

Eight different TAs are observed interacting with students in the videotapes. Six of the eight TAs observed were graduate students in University of Maryland Department of Phys- 
TABLE I. Teaching assistant attributes, tutorial sections, and student groups. The numbers indicate how many weeks each TA was observed teaching the indicated section.

\begin{tabular}{|c|c|c|c|c|c|c|}
\hline \multirow[b]{3}{*}{ TA } & \multirow[b]{3}{*}{ TA attributes } & \multicolumn{5}{|c|}{ Section (A-D) and student group (color) } \\
\hline & & \multirow{2}{*}{$\frac{\mathrm{A}}{\text { Yellow }}$} & \multirow{2}{*}{$\frac{\text { B }}{\text { Orange }}$} & \multicolumn{2}{|c|}{$\mathrm{C}$} & \multirow{2}{*}{$\frac{\mathrm{D}}{\text { Blue }}$} \\
\hline & & & & Green & Red & \\
\hline Stuart & Physics undergrad. & 7 & & & & \\
\hline Jim & Physics grad. student, PERG & 7 & & 1 & & \\
\hline Peter & Physics grad. student, PERG & & 7 & & & \\
\hline Max & Physics grad. student & & 7 & 7 & 5 & \\
\hline Andrew & Postdoc., PERG & & & 6 & 5 & \\
\hline Jason & Physics grad. student, PERG & & & & & 5 \\
\hline Caroline & Physics grad. student, PERG & & & & & 7 \\
\hline Ramona & Physics grad. student, PERG & & & & & 2 \\
\hline
\end{tabular}

ics; the seventh was an undergraduate physics major and the eighth was a postdoctoral fellow, both in the same department. Six of the eight TAs, including the postdoctoral fellow, were members of the University of Maryland Physics Education Research Group. We represent the TAs with gendercorrect pseudonyms (Stuart, Jim, Peter, Max, Andrew, Jason, Caroline, and Ramona). Table I shows which TAs and groups were associated with each section, along with the number of weeks each TA was observed teaching the indicated section. ${ }^{17}$

The final data set consists of 33 hours of tutorial videotape, documenting five different groups of students in four different sections working on seven different tutorials. In selecting our data, we did not attempt to create a random or representative set of videotapes. Instead, our data set represents a "best-case scenario:" a well-functioning class with experienced TAs, a supportive instructor, well-tested tutorials, and relatively engaged students.

\section{METHOD}

For each videotape in our study, we fast-forwarded through the videotape and paused when we observed a student-TA interaction occurring. We then observed the beginning of each interaction between students and TAs and classified that beginning as one of the following types:

Student initiated $(S)$. The students call a TA over for assistance or start a discussion with a TA who is already nearby.

TA initiated $(T)$. The TA arrives at the table spontaneously and starts a conversation.

Worksheet initiated $(W)$. The students reach a point in the worksheet that requests they discuss their results with a TA before proceeding (a "checkpoint"), and they call a TA over for the purpose of that discussion.

During coding, we made the following decisions.

(a) We prioritized verbal interactions over events in which the TAs placed themselves in physical proximity to the students. For example, if the TA came over and stood at a table but said nothing and then left, no interaction was recorded. If the TA came over and stood at a table and then a student asked the TA a question, the interaction was coded as student initiated.

(b) We did not use the substance of the conversation between the students and the TA to classify the interaction. For example, if the students called the TA over for specific assistance and the TA eventually included the checkpoint as part of the ensuing discussion, the interaction was still classified as student initiated, not worksheet initiated. This decision reflects our current focus on the initiation of the interaction, rather than its content.

(c) We did not use nonverbal signals between the students and the TA to classify the interaction. For example, if the TA came over and inquired whether the students were ready for a discussion, the interaction was classified as TA initiated, even though the students may possibly have summoned the TA to the table by eye contact. We found it difficult to observe nonverbal signals since the camera did not record the TA until he or she arrived at the table.

The analysis described above focused our attention on the initiation of the interactions between TAs and students in tutorial. We did not attempt to capture other properties of the interaction, such as its content, quality, length, desirability, effect, etc. We are extremely interested in those other properties and plan to investigate them in future research.

Inter-rater reliability was assessed among the three researchers responsible for classifying interactions. We checked for agreement along two measures: (1) the presence of an interaction and (2) the type of interaction (given agreement on its presence). On the first measure, inter-rater reliability was $76 \%$ before discussion. The low agreement resulted primarily from our analysis method: in fastforwarding through the tapes to identify interactions, very short interactions were sometimes missed because they occurred too quickly to cue the researcher to stop the tape. Interactions in which students spoke to an off-camera TA also tended to be missed because they were cued only by the posture of the students, not by the visual presence of a TA. When there was agreement on the presence of an interaction, inter-rater reliability on the type of interaction was $91 \%$. Differences resulted mostly from sound quality where the initia- 


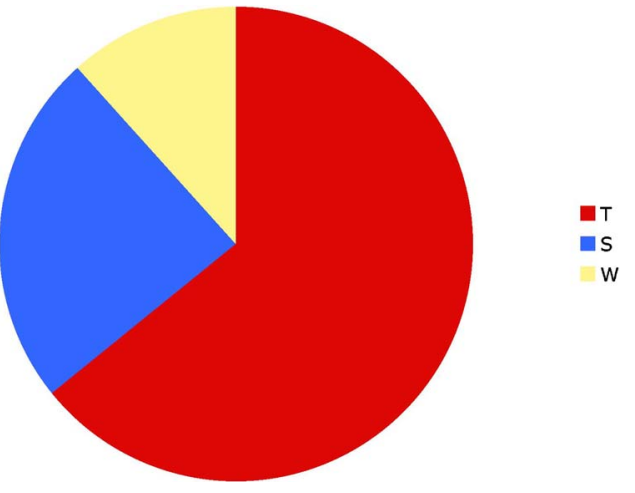

FIG. 1. (Color) Overall proportions of student-TA interactions that were (T) TA initiated, (S) student initiated, and (W) worksheet initiated.

tor of the interaction was difficult to hear or decipher. After discussion, the combined inter-rater reliability on both of these measures was $97 \%$.

\section{RESULTS}

This section presents the results of our analysis. First we present the overall proportion of the interactions of each type. Next, we examine the extent to which the overall interaction pattern is echoed by different student groups, different TAs, or different tutorial activities. Finally, we investigate the extent to which the interaction pattern appears to be determined by the student group or the TA.

\section{A. Overall results}

212 TA-student interactions were observed. Of these, 136 were TA initiated $(64 \%), 51$ were student initiated $(24 \%)$, and $25(12 \%)$ were worksheet initiated. Figure 1 illustrates these proportions.

\section{B. Comparison of interaction patterns for different subsets of the data}

In this section we explore the possibility that different subsets of the data show different initiation patterns. For example, if group characteristics play an important role in who initiates tutorial interactions, different groups might show different patterns of initiation. Similarly, TAs might have different interaction styles, or different tutorial activities might inspire different interaction patterns. We present each of these comparisons.

\section{Comparison of student groups}

Five different student groups were observed. Figure 2 shows the average number of interactions of each type that each group experienced per hour of tutorial. ${ }^{18}$ For every group there were more TA-initiated interactions than any other kind.

\section{Comparison of TAs}

Eight different TAs were observed interacting with student groups. Figure 3 shows the interaction pattern of each

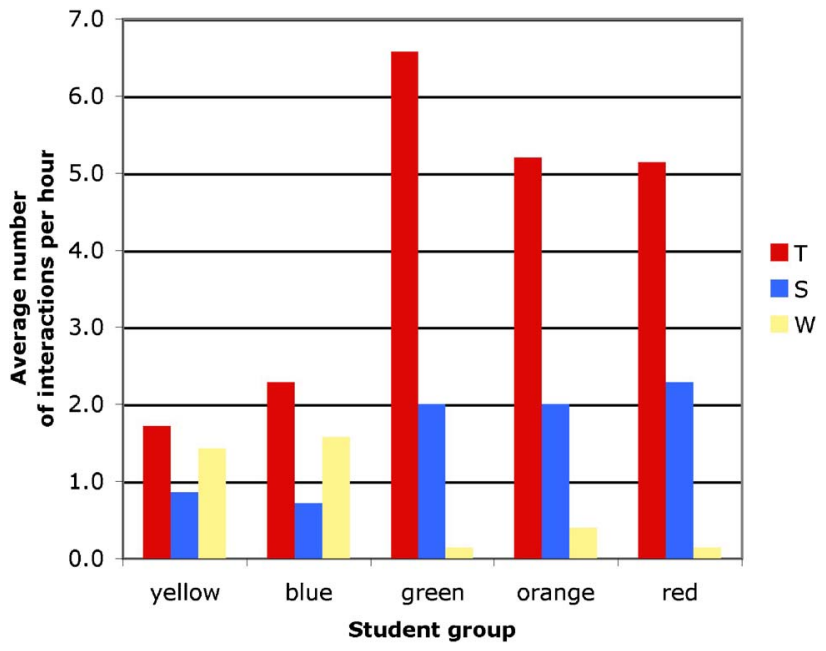

FIG. 2. (Color) Average number of interactions of each type that each student group experienced per hour of tutorial.

TA. ${ }^{19}$ There is some diversity of interaction patterns among the TAs; for example, Max and Andrew have a larger average number of TA-initiated interactions than the rest, while Stuart's interactions are less frequent overall and relatively equally distributed among the three types. However, six of the eight TAs have, on average, more TA-initiated interactions than any other kind.

Figure 3 reveals some similarities among TAs' interaction patterns. The similarities are not surprising, especially since novice TAs typically shared sections with TAs that were more experienced, and may well have learned an interaction style from them. Mentoring could explain the similarity between Jim's and Stuart's interaction patterns, and between Andrew's and Max's. Jason and Caroline, however, also had a mentoring relationship but had very different interaction patterns.

Because Max and Andrew interacted frequently with students and were observed with multiple groups, their interactions are a large fraction $(65 \%)$ of the total number of initia-

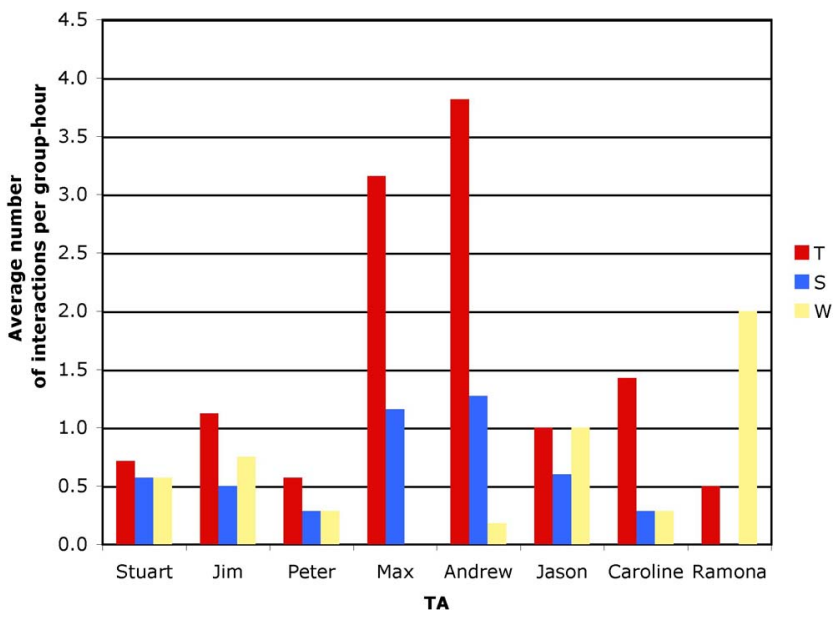

FIG. 3. (Color) Average number of interactions of each type that each TA experienced per "group-hour" of tutorial. ("Group-hours" is the total number of hours that a TA spent with the groups observed.) 
TABLE II. Tutorial topics.

\begin{tabular}{cc}
\hline \hline Tutorial & Physics topic \\
\hline 1 & Velocity graphs \\
2 & Acceleration graphs \\
3 & Newton's third law \\
4 & Newton's second law \\
5 & Free-body diagrams \\
6 & Momentum \\
7 & Work-energy \\
\hline \hline
\end{tabular}

tions observed. Although this contribution is large, we don't know whether Max and Andrew's interaction style is common or uncommon, and so we have not removed them from the data set.

\section{Comparison of tutorials}

Seven tutorials were observed for this study. Their topics are shown in Table II. Most of the tutorials were exclusively paper-and-pencil exercises; the exceptions were the first two, in which the students made extensive use of motion-detector laboratory equipment. ${ }^{20}$ Tutorial 3 (Newton's third law) included a demonstration, but the students did not handle the equipment.

Figure 4 shows the interaction pattern for each tutorial. ${ }^{21}$ TA-initiated interactions are the most common type for every tutorial.

Student-initiated interactions are more common for the first four tutorials and less common for the next three. Informal observations indicate that many of the student-initiated interactions in the first two weeks were equipment related. It might be that some tutorials are easier than others, inspiring fewer student-initiated interactions; it might be that students lose interest over the course of the semester; or there might be some other explanation. The small numbers of student- initiated interactions in any tutorial would make these questions difficult to study more rigorously.

No tutorial had an average of more than one worksheetinitiated interaction per group, in spite of the fact that most tutorials had at least two explicit checkpoints. ${ }^{22}$ This does not mean that the checkpoints were not discussed; it may be that the content of a checkpoint was covered in an interaction initiated by the TA (or the students). However, the absence of worksheet-initiated interactions calls the function of explicit checkpoints into question. If the checkpoints are not causing student-TA interactions to occur, we should perhaps articulate the benefit, if any, of their explicit appearance on the worksheets.

\section{Factors determining the initiation pattern}

The pattern of initiations that student groups and TAs experienced might be attributable to either characteristics of the group or characteristics of the TAs that group interacted with. For example, it could be the case that the orange, red, and green groups shared some characteristic that brought TAs to their tables frequently. Another possibility is that the TAs for those groups had a teaching style that propelled them to initiate a large number of interactions. In this section we investigate the extent to which either the student groups or the TAs seem to determine the interaction pattern.

\section{Investigating the extent to which interaction patterns are determined by student groups}

If the student group primarily determines the interaction pattern, then a group should show a similar interaction pattern when interacting with different TAs. Figure 5 shows (a) the yellow group's interactions with Stuart and Jim, (b) the blue group's interactions with Jason, Caroline, and Ramona, (c) the green group's interactions with Max, Andrew, and Jim, (d) the orange group's interactions with Max and Peter, and (e) the red group's interactions with Max and Andrew. The patterns for the orange and blue groups are very differ-

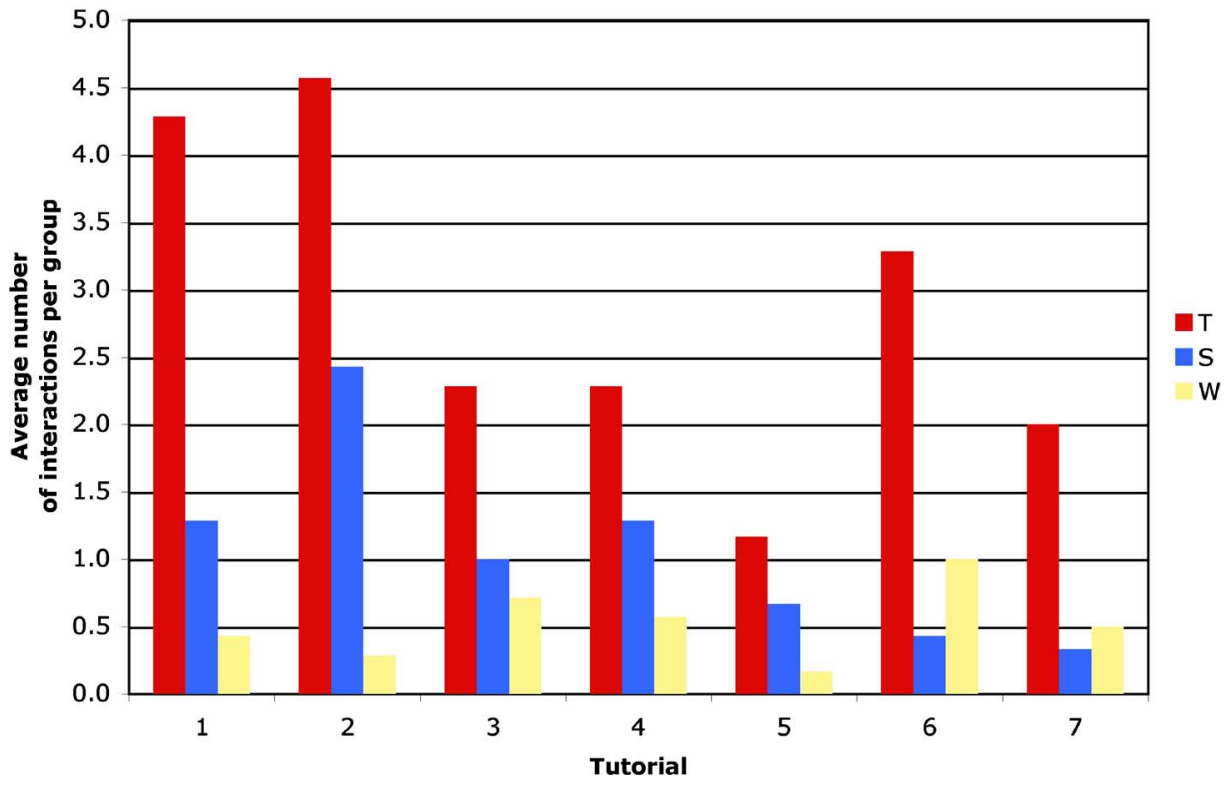

FIG. 4. (Color) Average number of interactions of each type that occurred per group for each tutorial. 


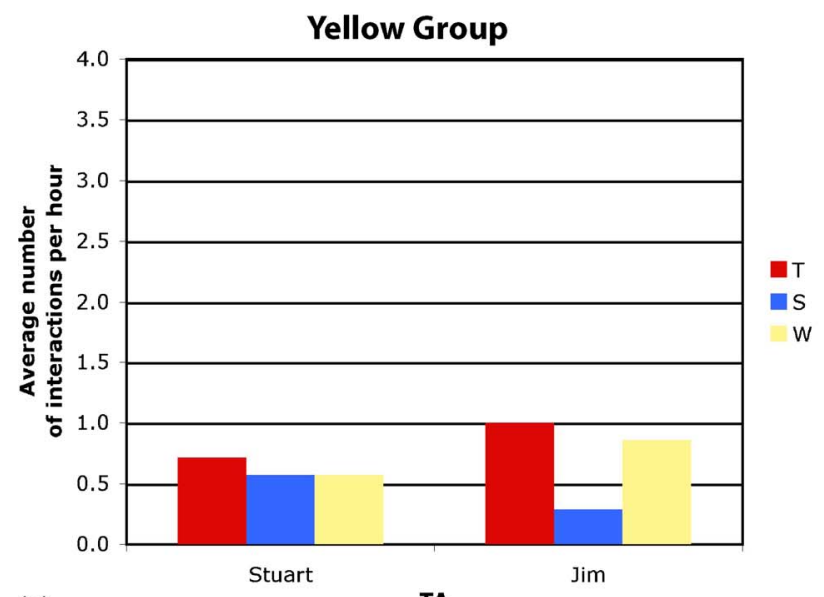

(a)

TA

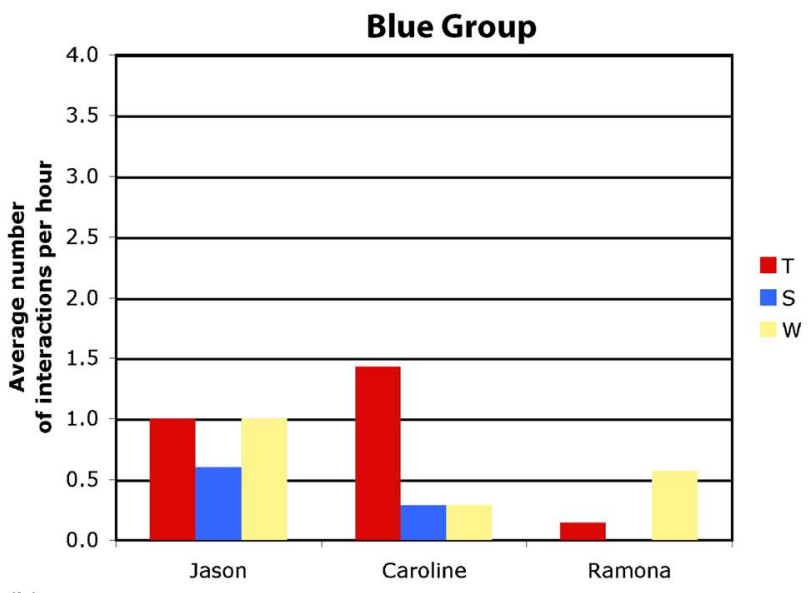

(b)

TA

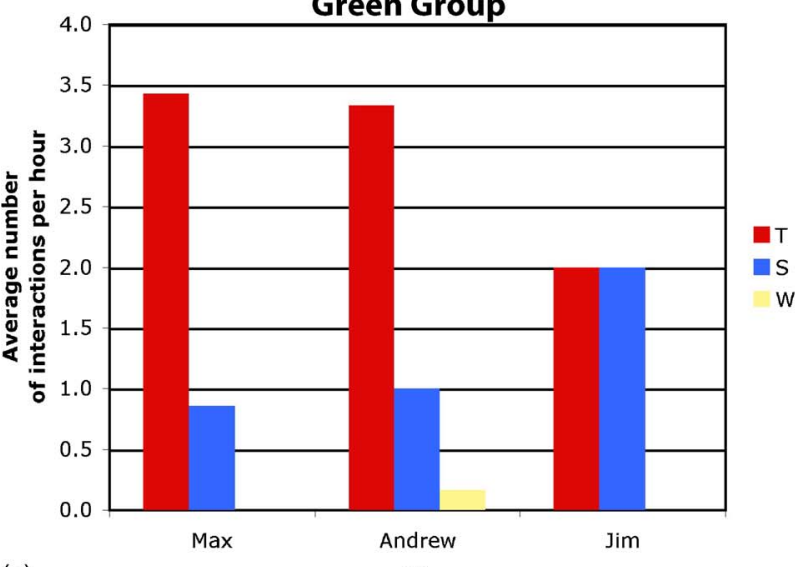

(c)

TA

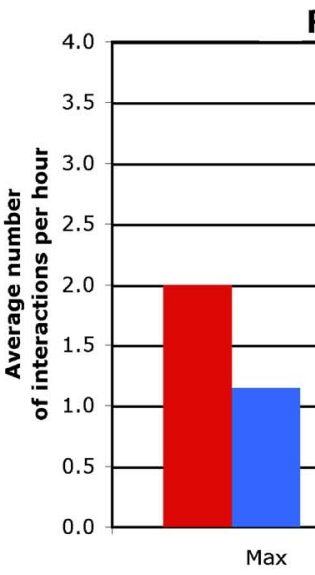

Red Group

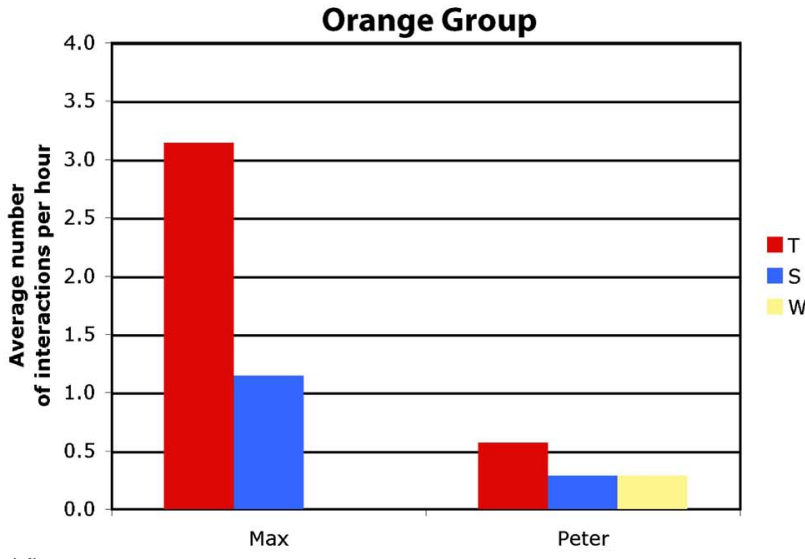

(d)

TA

(e)

TA

FIG. 5. (Color) (a) The yellow group's interactions with Stuart and Jim. (b) The blue group's interactions with Jason, Caroline, and Ramona. (c) The green group's interactions with Max, Andrew, and Jim. (d) The orange group's interactions with Max and Peter. (e) The red group's interactions with Max and Andrew.

ent for different TAs, suggesting that group characteristics do not determine the interaction pattern. The patterns for the green group were different for one TA (Jim) than for the two others (Max and Andrew), but the two others were similar to one another. The patterns for the yellow and red groups are fairly similar for the different TAs, suggesting either that those groups determine the interaction pattern, or that the TAs with whom they interacted were not significantly different as interactors even though they were different people. We investigate the possible similarity between the TAs in the next section.

\section{Investigating the extent to which interaction patterns are determined by TAs}

To determine the extent to which the interaction pattern is determined by TA characteristics, we observe different groups' interaction with the same TA. Figure 6 shows (a) the green, orange, and red groups' interactions with Max, and (b) 

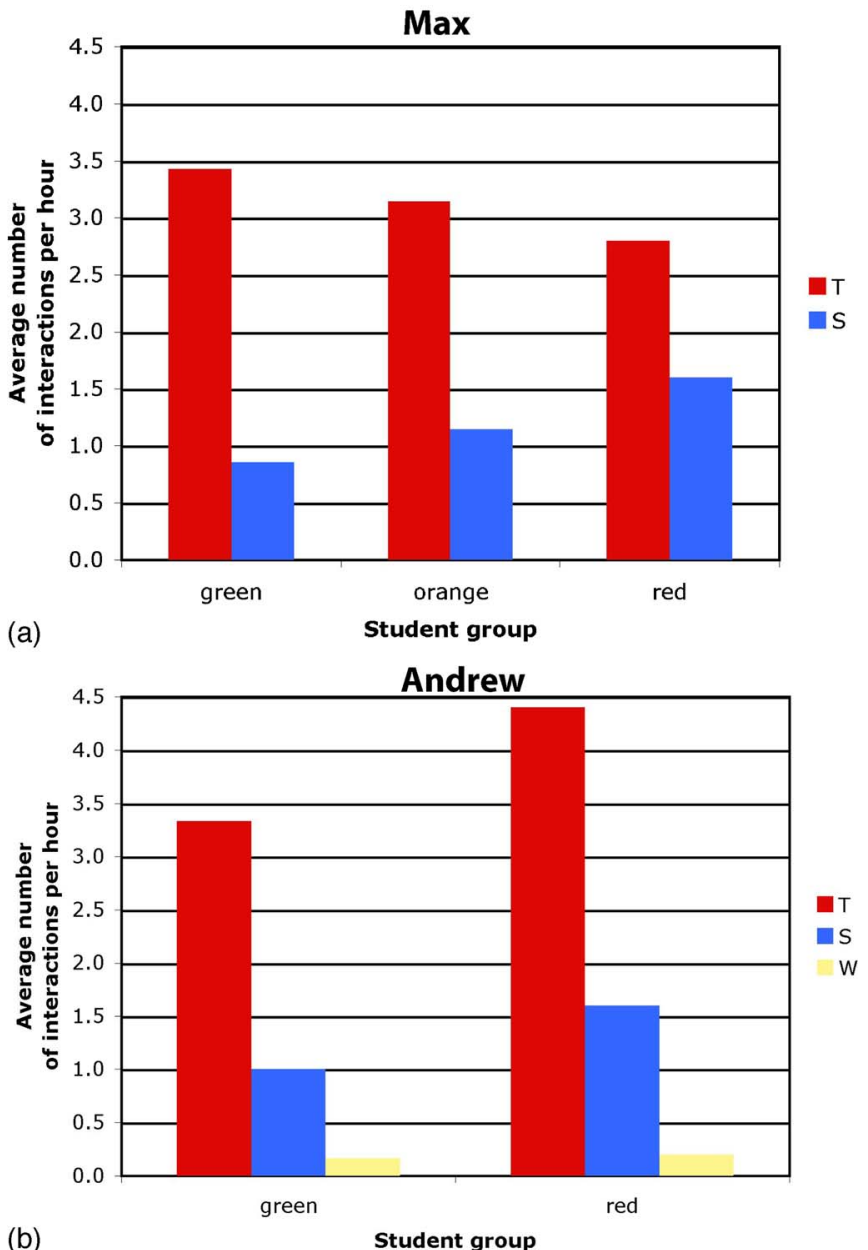

FIG. 6. (Color) (a) Max's interactions with the green, orange, and red student groups. (b) Andrew's interactions with the green and red student groups.

the green and red groups' interactions with Andrew. The patterns are similar for the different student groups, suggesting either that the interaction pattern is primarily determined by the TA, or that the green, orange, and red groups are themselves similar. The latter hypothesis is weakened by the fact that the green and red groups had worksheet-initiated interactions with Andrew but not with Max. Further, in Fig. 5(d), the orange group is shown to have a very different interaction pattern with Max than with Peter, which is evidence that the TA influences the pattern. Our tentative conclusion is thus that the interaction pattern is mostly determined by the TA. ${ }^{23}$ The fact that the majority of the interactions are TA initiated further supports this result.

\section{SUMMARY}

Our study shows that most student-TA interactions in tutorial are TA initiated, meaning they probably have at least the initial purpose of satisfying the TA. This result applies (on average) to all student groups observed, all tutorials observed, and nearly all TAs in the study. Preliminary analysis suggests that this interaction pattern is mainly determined by the TA rather than by the students (which is not surprising, given that the TAs initiate most of the interactions). Studentinitiated interactions are comparatively rare (on average) for all groups, all tutorials, and most TAs. Worksheet-initiated interactions are even more rare overall, although they outnumber student-initiated interactions for a few groups, some TAs, and a few tutorials.

More generally, our study demonstrated to us that, in spite of our long experience as tutorial instructors and developers, our sense of what occurs in the tutorial classroom is not necessarily accurate. We need systematic investigationsboth qualitative and quantitative-to learn what is taking place in our research-based instructional environments.

\section{DISCUSSION}

Our analysis does not address whether the observed pattern of student-TA interactions is desirable or not. However, our initial response is to be concerned. Our intention is for tutorials to be an environment in which students are primarily responsible for their own learning, and a wealth of student-initiated interactions would provide some evidence of such an environment. ${ }^{7-11}$ Our results, in contrast, seem to suggest that the TAs are driving the tutorial activity. In the absence of many student-initiated interactions, we wonder whether students are willing to ask questions, whether they feel that their own ideas are worth spontaneously discussing with a TA, or whether they are reluctant to make any effort over the minimum required of them. We also wonder whether student-TA interactions address issues relevant to the students, or whether well-intentioned TAs are focusing on issues that they judge to be important. Further, the absence of worksheet-initiated interactions causes us to worry that students are not taking the worksheets seriously.

It may be that our concerns are unfounded. Students may be perfectly ready to initiate interactions, but find they do not need to because a TA appears at their table at just the right time. TAs may be initiating interactions in which they sensitively detect and respond to student thinking. Students may be discussing the checkpoints in depth during TA-initiated interactions. TA-initiated interactions may even be more desirable than student-initiated interactions, if the TAs are better than the students at bringing important issues to the fore. None of these possibilities are ruled out by the present analysis, and we plan for future analyses to probe these alternatives.

Another consideration is whether it is the relative proportion or the number of TA- and student-initiated interactions that concerns us. It is tempting to think that more TAinitiated interactions would mean fewer student-initiated interactions - that the TAs "crowd out" the students due to the finite time in the tutorial-but in our examples, TAs who initiated more interactions (Max and Andrew) also experienced more student-initiated interactions. Future research will investigate the quantity of student-TA interactions and how that varies by TA, group, and tutorial activity.

Whether or not the current pattern is desirable or not in terms of student learning, our results offer guidance important to TA professional development. Apparently, most TAs do not tend to wait for students (or the worksheet) to initiate 
interactions. Supervisors who want to see many studentinitiated interactions in tutorial may want to help TAs hold back and encourage students to take the lead. Those who want to build on TAs' natural strengths may want to focus on helping TAs initiate interactions well (rather than trying to work against their tendency to initiate). Whatever our emphasis, we should not typically expect that students will be inundating TAs with spontaneous questions.

\section{QUESTIONS FOR FUTURE RESEARCH}

This study raises many questions for future research. Some have already been suggested in the above discussion: Are students willing (and do they have the opportunity) to ask spontaneous questions in tutorial? Are student-TA interactions centered on issues relevant to the students? Are checkpoints addressed even in the absence of worksheetinitiated interactions? Other questions might probe more deeply the meaning of the results presented in this paper. Why are the TAs initiating the majority of the interactions? Do they feel obliged to do so? Are they otherwise unoccu- pied? Do they perceive the students as needing assistance whenever they do not have the right answer (which students often do not, especially while a tutorial is in progress)? Do the students benefit from TA assistance, or are the TAs interrupting high-quality interactions within the student group? Yet other questions have been suggested by informal observations made during our analysis. It appears, for example, that early tutorials have many, short, equipment-related interactions; that later tutorials have fewer, longer interactions; and that discussions of conceptual physics issues are rare. How many interactions are there per tutorial, what is their content, and how long do they last? We look forward to investigating these questions in future research.

\section{ACKNOWLEDGMENTS}

We are grateful to Andrew Elby, M. Saalih Allie, David Hammer, E. F. Redish, and the members of the Physics Education Research Group at the University of Maryland for substantive discussions of this research. This work was supported in part by the National Science Foundation Grants No. REC 0440113 and No. REC 0529482.
${ }^{1}$ Other models of small-group, active-engagement, worksheetbased learning include, for example, D. Hammer, Discovery learning and discovery teaching, Cogn. Instruct. 15, 4 (1997).

${ }^{2}$ E. F. Redish, D. Hammer, and A. Elby, NSF REC 0087519, 2001-2005 (unpublished).

${ }^{3}$ How People Learn: Brain, Mind, Experience, and School, edited by J. D. Bransford, A. L. Brown, and R. R. Cocking (National Academy Press, Washington, DC, 2000).

${ }^{4}$ L. C. McDermott, P. S. Shaffer, and the Physics Education Group at the University of Washington, Tutorials in Introductory Physics (Prentice-Hall, Englewood Cliffs, NJ, 1998); P. S. Shaffer and L. C. McDermott, Research as a guide for curriculum development: An example from introductory electricity. Part II: Design of instructional strategies, Am. J. Phys. 60, 11 (1992); L. C. McDermott, P. S. Shaffer, and M. Somers, Research as a guide for curriculum development: An illustration in the context of the Atwood's machine, ibid. 62, 1 (1994).

${ }^{5}$ N. D. Finkelstein and S. J. Pollock, Replicating and understanding successful innovations: Implementing tutorials in introductory physics, Phys. Rev. ST Phys. Educ. Res. 1, 010101 (2005).

${ }^{6}$ See Refs. 1-5,12,15; R. Hake, Interactive-engagement versus traditional methods: A six-thousand-student survey of mechanics test data for introductory physics courses, Am. J. Phys. 66, 64 (1998); E. F. Redish, Implications of cognitive studies for teaching physics, ibid. 62, 796 (1994).

${ }^{7}$ Strategic Help-Seeking: Implications for Learning and Teaching, edited by S. A. Karabenick (Lawrence Erlebaum, Hillsdale, NJ, 1998).

${ }^{8}$ Help Seeking in Academic Settings: Goals, Groups, and Contexts, edited by S. A. Karabenick and R. S. Newman (Lawrence Erlebaum, Hillsdale, NJ, 2006).

${ }^{9}$ S. A. Karabenick and R. Sharma, in Student Motivation, Cognition, and Learning: Essays in Honor of Wilbert J. McKeachie, edited by P. Pintrich, D. Brown, and C. E. Weinstein (Lawrence
Erlebaum, Hillsdale, NJ, 1994).

${ }^{10}$ A. Arbreton, in Strategic Help-Seeking: Implications for Learning and Teaching (Ref. 7), Chap. 5.

${ }^{11}$ R. Ames, in New Directions in Helping: Vol. 2. Help Seeking, B. M. DePaulo, A. Nadler, and J. D. Fisher (Academic Press, New York, 1983).

${ }^{12}$ E. F. Redish, J. Saul, and R. N. Steinberg, On the effectiveness of active-engagement microcomputer-based laboratories, Am. J. Phys. 65, 45 (1997); E. F. Redish and R. N. Steinberg, Teaching physics: Figuring out what works, Phys. Today 52 (1), 24 (1997).

${ }^{13}$ For descriptions of the use of tutorials, see Refs. 4,5,12.

${ }^{14}$ A. Elby, R. E. Scherr, D. Hammer, S. Vokos, and S. Rosenberg, NSF CCLI 0341447, 2004-2006 (unpublished).

${ }^{15}$ A. Elby, Helping physics students learn about learning, Am. J. Phys. 69, S54 (2001).

${ }^{16}$ These groups were not the only "watchable" groups; some videotaped groups were not classified. The groups included average, above-average, and below-average students as measured by their overall performance in the course. "Watchable" groups were not typically better than average or more successful in mastering the conceptual content of the tutorials, except to the extent that ontask groups tend to do better than off-task groups.

${ }^{17}$ That number may be different from the number of tutorials (seven) due to missed weeks, substitutions, or (in the case of the red group) missing videotapes.

${ }^{18}$ The averaging is necessary because for one group (red), only five tutorials are documented, rather than seven.

${ }^{19}$ Since not all TAs were observed for the same number of hours, and different TAs served different numbers of groups, the figure presents each type of interaction averaged over the number of "group-hours" observed for that TA. "Group-hours" is the total number of hours that a TA spent with the groups observed, 
i.e., the sum of each row in Table I.

${ }^{20}$ R. K. Thornton and D. R. Sokoloff, Learning motion concepts using real-time microcomputer-based laboratory tools, Am. J. Phys. 58, 858 (1990).

${ }^{21}$ Since not all tutorials were observed for the same number of groups, the figure presents the number of interactions of each type averaged over the number of groups that were observed doing that tutorial.

${ }^{22}$ Tutorial 1 had one explicit checkpoint, tutorials 2-4 had two each, tutorials 5 and 6 had three each, and tutorial 7 had none.

${ }^{23}$ The number of interactions is too small to support a statistical analysis. 